\title{
Analysis of national trends in neurosurgical resident attrition
}

\author{
Nitin Agarwal, MD, ${ }^{1}$ Michael D. White, BS, ${ }^{1}$ Susan C. Pannullo, MD, ${ }^{2}$ and Lola B. Chambless, MD $^{3}$ \\ ${ }^{1}$ Department of Neurological Surgery, University of Pittsburgh Medical Center, Pittsburgh, Pennsylvania; ${ }^{2}$ Department of \\ Neurological Surgery, Weill Cornell Medicine, New York, New York; and ${ }^{3}$ Department of Neurological Surgery, Vanderbilt \\ University Medical Center, Nashville, Tennessee
}

OBJECTIVE Resident attrition creates a profound burden on trainees and residency programs. This study aims to analyze trends in resident attrition in neurological surgery.

METHODS This study followed a cohort of 1275 residents who started neurosurgical residency from 2005 to 2010. Data obtained from the American Association of Neurological Surgeons (AANS) included residents who matched in neurosurgery during this time. Residents who did not finish their residency training at the program in which they started were placed into the attrition group. Residents in the attrition group were characterized by one of five outcomes: transferred neurosurgery programs; transferred to a different specialty; left clinical medicine; deceased; or unknown. A thorough internet search was conducted for residents who did not complete their training at their first neurosurgical program. Variables leading to attrition were also analyzed, including age, sex, presence of advanced degree (Ph.D.), postgraduate year (PGY), and geographical region of program.

RESULTS Residents starting neurosurgical residency from 2005 to 2010 had an overall attrition rate of $10.98 \%$. There was no statistically significant difference in attrition rates among the years $(p=0.337)$. The outcomes for residents in the attrition group were found to be as follows: $33.61 \%$ transferred neurosurgical programs, $56.30 \%$ transferred to a different medical specialty, $8.40 \%$ left clinical medicine, and $1.68 \%$ were deceased. It was observed that women had a higher attrition rate (18.50\%) than men (10.35\%). Most attrition (65.07\%) occurred during PGY 1 or 2 . The attrition group was also observed to be significantly older at the beginning of residency training, with a mean of 31.69 years of age compared to 29.31 in the nonattrition group $(p<0.001)$. No significant difference was observed in the attrition rates for residents with a Ph.D. $(9.86 \%)$ compared to those without a Ph.D. $(p=0.472)$.

CONCLUSIONS A majority of residents in the attrition group pursued training in different medical specialties, most commonly neurology, radiology, and anesthesiology. Factors associated with an increased rate of attrition were older age at the beginning of residency, female sex, and junior resident (PGY-1 to PGY-2). Resident attrition remains a significant problem within neurosurgical training, and future studies should focus on targeted interventions to identify individuals at risk to help them succeed in their medical careers.

https://thejns.org/doi/abs/10.3171/2018.5.JNS18519

KEYWORDS residency; resident attrition; neurological surgery; burnout

$\mathrm{N}$ EUROSURGICAL residency is demanding and strenuous. Long and unpredictable hours, arduous surgeries, and managing medically complex patients can lead to burnout. ${ }^{2}$ To perform at a high level during neurosurgical residency, one must be able to overcome and manage the profound stressors associated with the profession. Residents who are ultimately unsuccessful in completing neurosurgical training face significant opportunity costs, and their programs and co-residents share this burden. Thus, minimizing resident attrition is an important goal of any successful neurosurgical training program.

The unique and unforgiving lifestyle of surgery is known to lead to high rates of resident burnout. Attrition of peers interferes with the flow of residency training, which diminishes resources and adds additional challenges for the department to overcome in order to run efficiently. ${ }^{14}$ Resident attrition has been well-studied in general surgery, where attrition rates are believed to be some of the highest of any medical specialty. It has been reported

ABBREVIATIONS ACGME = Accreditation Council for Graduate Medical Education; PGY = postgraduate year; ROC = receiver operating characteristic. SUBMITTED February 25, 2018. ACCEPTED May 29, 2018.

INCLUDE WHEN CITING Published online November 23, 2018; DOI: 10.3171/2018.5.JNS18519. 


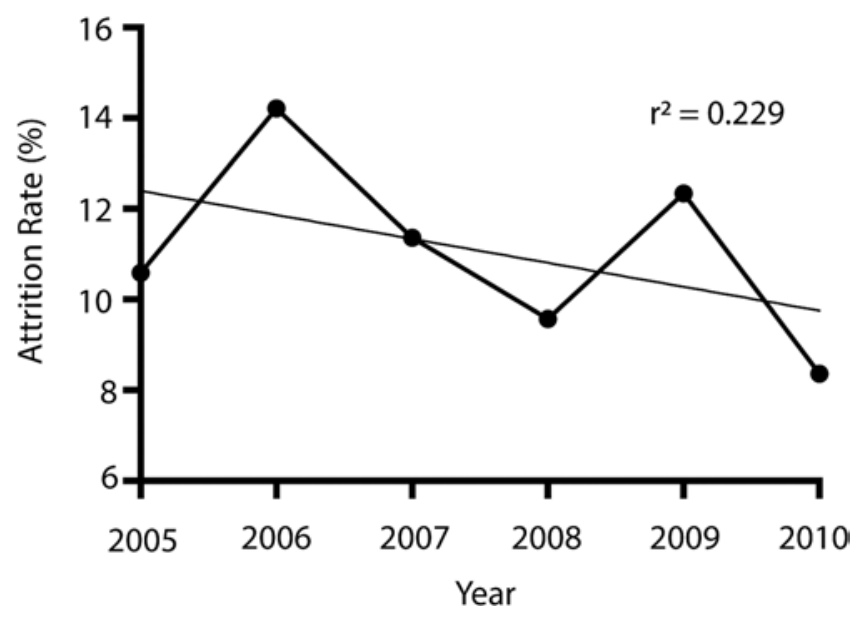

FIG. 1. Graph showing the resident attrition rate for each class of residents matching into neurosurgery between 2005 and 2010. There was no trend in the change of attrition rate between years $\left(r^{2}=0.229, p=\right.$ 0.337).

that general surgery attrition rates can vary between $17 \%$ and $26 \%$. $^{6,11,12}$ In an attempt to improve resident lifestyle and patient safety, the Accreditation Council for Graduate Medical Education (ACGME) implemented duty hour restrictions in 2003 and 2011, limiting residents to an 80hour work week and maximum shift hours. Previous research demonstrates that duty hour restrictions, however, did not result in any appreciable decrease in attrition. ${ }^{13}$ In fact, Everett et al. reported that attrition rates in general surgery were slightly higher after the incorporation of duty hour restrictions. ${ }^{8}$

Resident attrition has been reported to be associated with a plethora of factors, and much variation is reported in the literature as to what leads to this phenomenon, including lifestyle, postgraduate year (PGY)-1 status, nonsouthern programs, and the perception that clinical duties were prioritized over education. ${ }^{5,6,22}$ Although resident attrition has been heavily studied in other medical specialties, there is a lack of investigation pertaining specifically to neurosurgical training, which is uniquely lengthy and specialized. In this paper we present a longitudinal survey of the national trends of resident attrition for neurosurgical residencies for trainees entering the field from 2005 to 2010.

\section{Methods}

Prospectively collected data from the American Association of Neurological Surgeons (AANS) was retrospectively reviewed. The AANS reports included every resident who matched into a neurosurgical residency program from 2005 to 2017, along with their degrees, which program they matched into, whether they transferred into a different program, start and end dates at each program, birth date, and deceased date (if applicable). Using this database, neurosurgery residents who started residency from 2005 to 2010 were included in the study cohort and analyzed for whether they finished their respective programs. Residents who did not complete their full residency training were then placed into 5 outcome categories: transferred to a new neurosurgery program, transferred to a different specialty, left clinical medicine, deceased, or unknown. Placement in the "unknown" category indicates that information on these individuals could not be reliably confirmed via Internet search. Additionally, outcomes for residents with more than one instance of attrition were categorized based on their final outcome category. Variables leading to attrition were also analyzed, including age at the beginning of residency, sex, presence of a Ph.D., PGY in neurosurgery, and geographical location of residency program. For geographical location analysis, programs experiencing attrition were placed into one of four geographical groupings based on the US Census: Northeast, South, Midwest, and West (https://www2.census.gov/geo/ pdfs/maps-data/maps/reference/us_regdiv.pdf). SPSS software (IBM) was used to analyze statistical significance, including a linear regression analysis for change in attrition rate, Fisher's exact test for sex and advanced degrees, a 2-sample t-test and receiver operating characteristic (ROC) curve for age, and a chi-square goodness-of-fit test for program geographical location.

\section{Results}

\section{Attrition by Class}

For residents starting neurosurgical residency from 2005 to 2010, it was found that a total of 140 of 1275 residents who matched neurosurgery left their initial training program. The total attrition rate during this time period was $10.98 \%$. The highest rate of attrition was found for the class starting in 2006, with an attrition rate of $14.21 \%$. The class starting in 2010 had the lowest rate at $8.37 \%$ (Fig. 1). Nevertheless, a regression analysis demonstrated no significant change in attrition among years $\left(r^{2}=0.229\right.$, $\mathrm{p}=0.337)$.

\section{Transfers}

A majority of the residents leaving their programs transferred to different medical specialties outside of neurosurgery. Of the residents leaving a neurosurgery program, $33.61 \%$ transferred to another neurosurgery program, whereas $56.30 \%$ transferred into a different medical specialty. Besides neurosurgery, the three most common specialties residents transferred to were neurology (14.95\%), radiology (13.08\%), and anesthesiology (12.15\%; Fig. 2). In addition, $8.40 \%$ of residents leaving their initial neurosurgery program left clinical medicine altogether and $1.68 \%$ did not complete residency due to death (Fig. 3 ). The 10 residents who left clinical medicine altogether pursued a variety of different opportunities; these ranged from taking $\mathrm{CEO}$ positions to starting businesses. There were 5 residents with more than one instance of attrition. Of these residents, only 2 ended up finishing neurosurgical residency. Reliable information regarding the ultimate career choice for 21 of the 140 residents who left their initial program could not be verified via thorough an internet search, and were therefore marked as "unknown" and omitted from these values.

\section{Sex Differences}

A comparison of attrition rates was conducted between 


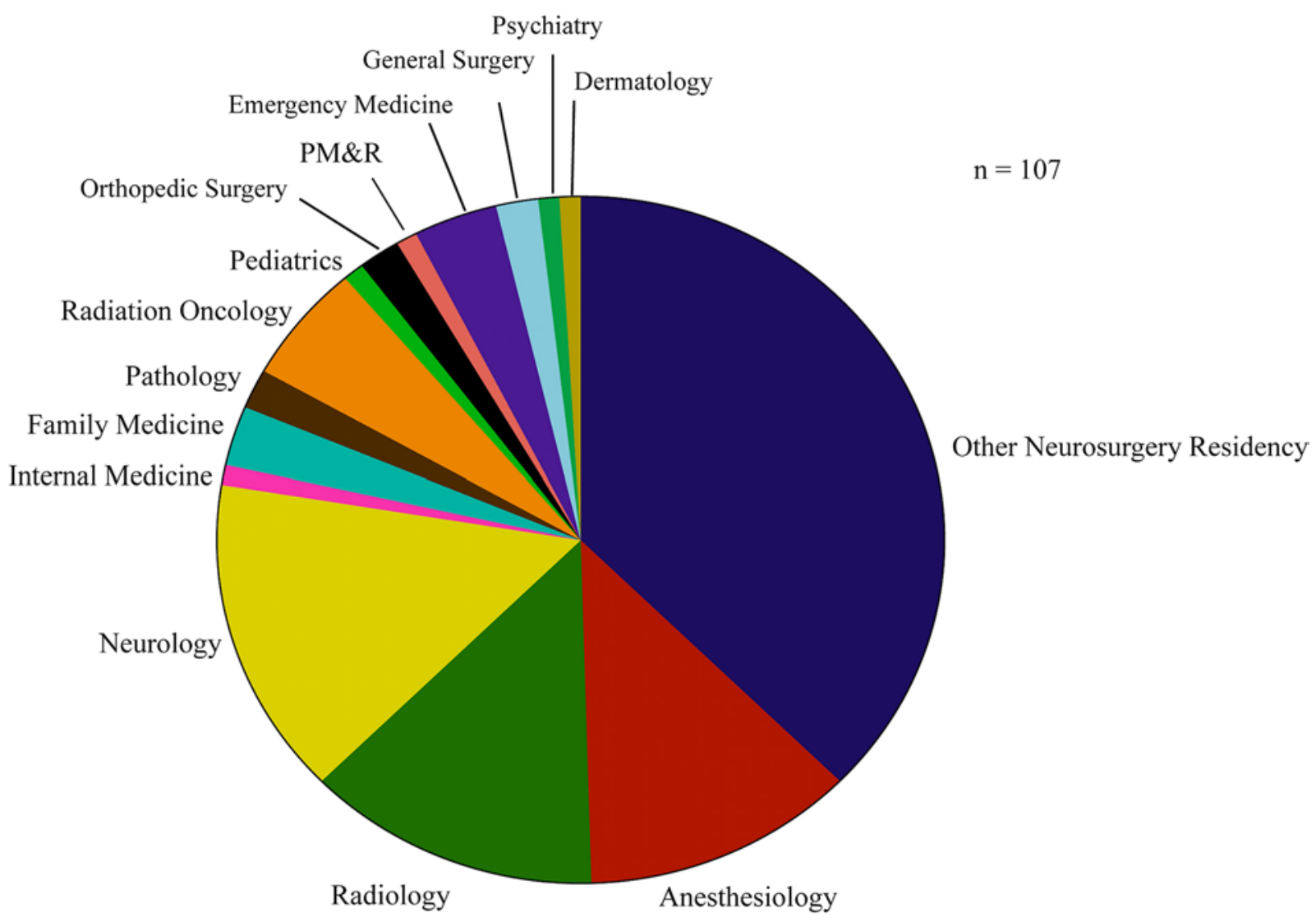

FIG. 2. Pie chart representing the different specialties transferring residents chose to pursue. The specialties for a total of 107 transferring residents were found. The percentage of transferring residents who went into each specialty were as follows: other neurosurgery residency $(37.38 \%)$, anesthesiology $(12.15 \%)$, radiology $(13.08 \%)$, neurology $(14.95 \%)$, internal medicine $(0.93 \%)$, family medicine $(2.80 \%)$, pathology $(1.87 \%)$, radiation oncology $(5.61 \%)$, pediatrics $(0.93 \%)$, orthopedic surgery $(1.87 \%)$, physical medicine and rehabilitation (PM\&R; 0.93\%), emergency medicine (3.74\%), general surgery (1.87\%), psychiatry $(0.93 \%)$, and dermatology $(0.93 \%)$. Figure is available in color online only.

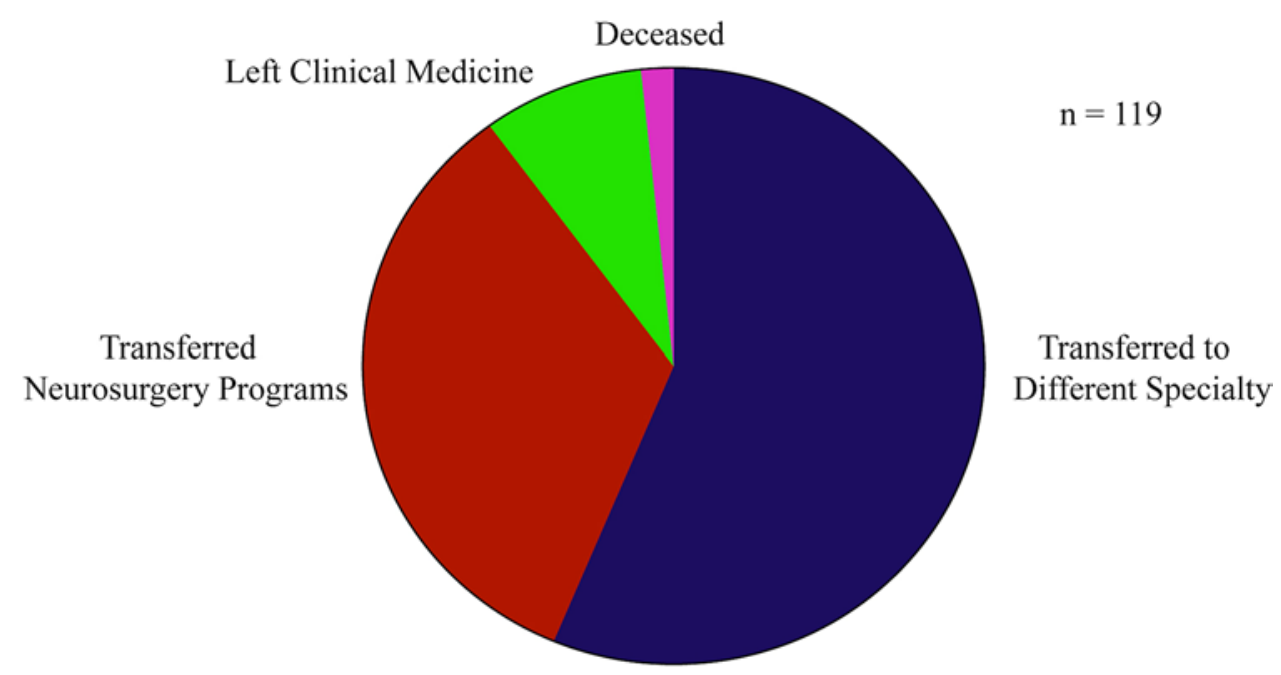

FIG. 3. One hundred nineteen residents in the attrition group with known outcomes were included in the figure. The 21 residents categorized as "unknown" were omitted. The percentage of residents within each outcome category was as follows: transferred neurosurgery programs $(33.61 \%)$, transferred to different specialty $(56.30 \%)$, left clinical medicine $(8.40 \%)$, and deceased $(1.68 \%)$. Figure is available in color online only. 


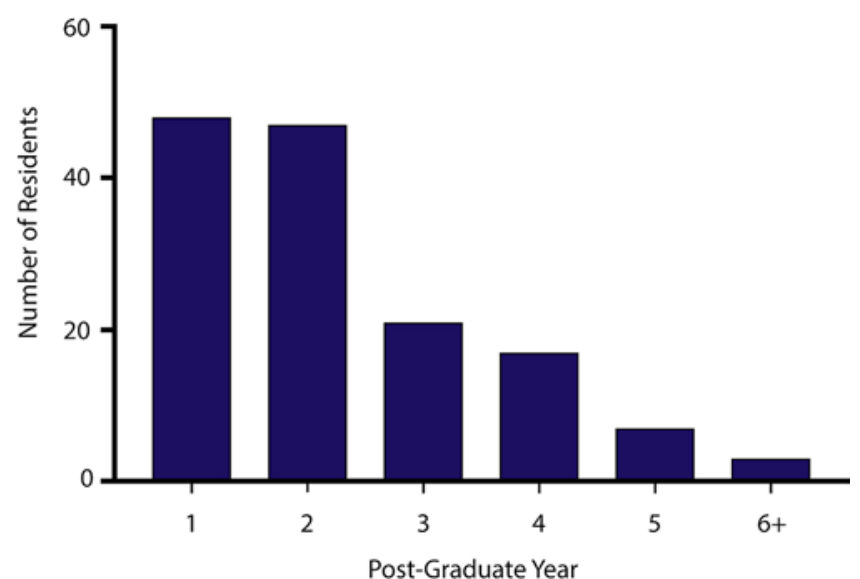

FIG. 4. Resident attrition categorized by PGY. This figure represents a total of 140 different residents leaving their original residency programs; however, there are 146 plotted points due to 5 residents leaving programs more than once. Breakdown of resident attrition by PGY includes: 48 PGY-1 residents, 47 PGY-2 residents, 21 PGY-3 residents, 18 PGY-4 residents, 8 PGY-5 residents, and 4 PGY-6 or above residents. Figure is available in color online only.

men and women to determine any difference due to sex. Between 2005 and 2010, there were a total of 1130 males and 173 females matching neurosurgery. Female attrition rate was found to be $18.50 \%$, compared to $10.35 \%$ for males $(\mathrm{p}<0.01)$. Of residents transferring, $18.19 \%$ of females transferred to another neurosurgery program, compared to $42.35 \%$ of males. Besides neurosurgery, the most frequent specialty females transferred to was neurology (27.27\%) and anesthesiology (22.72\%). The most frequent specialty for males, besides neurosurgery, was radiology $(14.12 \%)$ and neurology (11.76\%).

\section{Age Differences}

Age was found to be a significant factor in resident attrition. From the study cohort, age at the start of residency could be elucidated for 47 residents in the attrition group and 822 residents in the nonattrition group. The mean age at start of residency for the attrition group was 31.69 years, compared to 29.31 years among the nonattrition group. The difference between the two groups was observed to be 2.38 years ( $\mathrm{p}<0.001$; CI 1.44-3.32). Utilizing receiver operating characteristic (ROC) curve analysis, the optimal age cutoff for which residents were more likely to leave was found to be 30 years (sensitivity $=0.64$; specificity $=0.71$ )

\section{Other Factors}

Residents with both an M.D. and Ph.D. were not found to have significantly different rates of attrition. Of the 71 residents with a Ph.D., 7 of them left their original neurosurgery program. This resulted in an attrition rate of $9.86 \%$ for residents with a Ph.D., compared to $11.04 \%$ for residents without one $(\mathrm{p}=0.472)$.

Trends were also observed regarding the year in which residents left. Attrition occurred most often in the first and second postgraduate year. A majority of attrition (65.07\%) occurred during PGY-2 or before, and the number of resi- dents leaving decreased with each subsequent year (Fig. 4). It should be noted that although the total attrition group for this study has 140 residents, the attrition group for the PGY analysis contains 146 residents due to 5 residents transferring programs more than once.

Residents in the attrition group were also analyzed by residency. There were 146 cases of resident attrition and a total of 72 residency programs experiencing loss of a resident. Among residency programs with at least 3 instances of attrition, $45 \%$ were located in the South, $40 \%$ in the Midwest, $10 \%$ in the West, and 5\% in the Northeast ( $\mathrm{p}=$ 0.0186).

\section{Discussion}

Resident attrition is a salient issue in graduate medical education. Residents unsuccessful in their residency training impose high costs on themselves as well as on the programs at which they were training. From a monetary prospective, one study valued the annual cost of training a single resident at $\$ 141,240$ per year. ${ }^{23}$ The 140 residents observed to leave their residency program during the 5 -year period of this study theoretically cost the training hospitals more than $\$ 19$ million. This figure only increases for residents who leave after several years of training. This significant monetary cost to training hospitals is in addition to the personal costs imposed on residents, including relocating, finding another position/job, and adding mental stress. By identifying factors that lead to attrition, residency programs can identify at-risk individuals and help them to succeed in their medical careers.

The results of this study allow for characterization of variables relating to attrition in neurological surgery and overall trends observed from residents starting in 2005 to 2010. During this time period, there was an overall attrition rate of $10.98 \%$. The current literature has reported attrition rates both higher and lower than this percentage in various specialties. Specialties such as obstetrics and gynecology, anesthesiology, and neurology have reported considerably lower attrition rates of $4.2 \%, 3.6 \%$, and $2.9 \%$, respectively. ${ }^{10,15}$ Attrition rates among surgical specialties vary widely; otolaryngology and plastic surgery have reported rates of $1.2 \%$ and $3 \%$, respectively, whereas general surgery residencies have shown attrition rates ranging from $17 \%$ to $26 \%{ }^{6,11,12}$ In comparison to other specialties, neurosurgery was observed to have one of the highest rates of attrition, with only general surgery reporting higher values.

The most common cause for attrition in other surgical specialties such as general surgery is reported to be the uncontrollable lifestyle and inability to maintain a worklife balance. ${ }^{11,22}$ Sullivan et al. reported that up to $76 \%$ of attrition was caused by lifestyle. ${ }^{22}$ This conflict with maintaining an adequate work-life balance during surgical training is supported by our results. Over $65 \%$ of attrition was characterized by residents leaving the field of neurosurgery altogether. Additionally, all but 4 of the residents choosing other specialties ended up pursuing a nonsurgical specialty. The ACGME implemented an 80-hour work week restriction in 2003 and additional duty hour restrictions in 2011 in an attempt, in part, to reduce this strain 
on residents. Although we have not yet had a residency class to start and finish after the 2011 duty hour restrictions were implemented, some observations can still be made with the current data. Of the residency classes starting from 2005 to 2010, the 2010 class spent all but 1 year under the new 2011 duty hour restrictions and also had the lowest observed attrition rate at $8.37 \%$. Still, further study is required as there was no statistically significant difference in the change in attrition rates over time during the study period.

Although women only made up $15.31 \%$ of neurosurgical residents, they had a higher rate of attrition than their male counterparts. When separated by sex, men had an overall attrition rate of $10.35 \%$, compared to $18.50 \%$ for women. Similar results regarding higher rates of attrition for women have been reported in prior studies for neurosurgery, ${ }^{19}$ general surgery, ${ }^{11,16,22}$ and orthopedic surgery. ${ }^{3}$ The literature has documented the factors that affect surgical career choices for women. Among these factors are a lack of female role models in the field, perceived male bias, and sexual harassment., ${ }^{4,18,20,21}$ Of residents who transferred, $42.35 \%$ of men remained in a neurosurgical residency compared to only $18.19 \%$ of women. This indicates that attrition for women may not be due to problems confined to a certain program, but a dissatisfaction with the field of neurosurgery in general. It should be noted that not a single woman who transferred out of neurosurgery pursued a surgical specialty. The higher attrition rate for women is also not believed to be associated with dissatisfaction of the medical field in general, as there was no statistically significant difference between the percentage of men and women leaving clinical medicine. Overall, sex was found to be an influential factor on attrition in neurosurgical residents, which deserves further study.

Residents in the attrition group were found to be significantly older than those in the nonattrition group. This discrepancy in age is most likely multifactorial and cannot be accurately explained within the context of this study. However, increasing age as a significant factor for attrition has been reported in general surgery as well. In a multivariate study by Naylor et al., it was observed that of the academic, demographic, and social variables studied, an age greater than 29 years old at the start of residency was the most important factor in predicting attrition. ${ }^{17} \mathrm{Al}-$ though it is not exactly known why older age is a factor for attrition, it may be attributed to the greater emphasis placed on family and lifestyle as one gets older. As previously stated above, lifestyle tends to be one of the most influential drivers of attrition within surgical specialties, potentially becoming increasingly important among older residents.

Residents who obtained a Ph.D. prior to matching neurosurgery were not observed to have a significantly different rate of attrition than those without one. This is surprising, as a previous study by Andriole et al. demonstrated that residents with an M.D. and Ph.D. prior to general surgery residency were associated with higher rates of attrition. ${ }^{1}$ This study explained that residents with a Ph.D. did not have more dissatisfaction with their initial residency choice, but rather they were more likely to be able to successfully change residencies. The differences observed in this study and the aforementioned study may be due to inherent differences among the two specialties or among the types of students who pursue them. The same association between residents with a Ph.D. in general surgery may not be present within neurosurgery. Additionally, general surgery has a much higher attrition rate than neurosurgery, possibly leading to the observed results. It should also be noted that residents with a Ph.D. are inherently older, and this finding is counterintuitive in light of the observation noted above in which older age is associated with attrition. This may indicate that residents with a Ph.D. have a better sense of the realities of a neurosurgical residency and the type of lifestyle they are undertaking.

The results of this study aligned with those from previous studies determining PGY as a variable in attrition. Prior studies have also observed the highest rates of attrition during PGYs 1-3., 52 The observed attrition from residents during their first two PGYs may represent a disconnect between students' expectations of neurosurgical residency and their actual experience. Sullivan et al. analyzed a cohort of PGY-1 and PGY-2 general surgery residents to determine the attitudes of residents who left their surgical residency program. This study found that PGY-1 and PGY-2 residents in the attrition group reported concerns regarding the length of training required, personal sacrifice, and an unanticipated toll on their personal life..$^{22}$ The concerns expressed by these residents emulate a shifting paradigm in resident expectations regarding lifestyle and work-life balance. Medical student and resident concerns over lifestyle have been well characterized in the literature. ${ }^{7,9}$ Despite students reporting lifestyle and work hours as important considerations for career choice, attrition among residents is still associated with these factors. This may represent either an inadequate exposure to the realistic lifestyle of a neurosurgical resident or a lack of clarity regarding the challenges associated with the specialty. A study conducted at the University of Toronto offered first-year medical students a 2-week focused surgical experience for those interested in a surgical career. This study found that the course augmented students' understanding of neurosurgery and also decreased the overall interest in neurosurgery as a career. However, those who remained interested had a higher motivation to pursue neurosurgery. ${ }^{24}$ This type of immersive program may be useful in providing medical students a more comprehensive understanding of the neurosurgical specialty and lead to more realistic expectations among future residents.

The authors are mindful of the limitations of this study. During the time period of this retrospective study, there was variation in the classification of the internship between neurosurgery and general surgery. Some residents intending to pursue a neurosurgical residency would match into general surgery for their internship year; therefore, residents who left during the internship year in this case may not have been represented among the data. Additionally, information on residents who left their programs was obtained via internet search. Inaccuracies in online information regarding these individuals could not be verifiably discovered. It is also important to note that some instances of attrition may have been due to programmatic factors rather than resident factors. Examples include inadequate 
training, faculty attrition, programs on probation, and program directors leaving.

\section{Conclusions}

The residency attrition rate in neurosurgical training was found to be $10.98 \%$ with no significant change from 2005 to 2010 . This rate of attrition is relatively high when compared to other specialties, although lower than reported rates for general surgery. The most common reason for residents leaving their programs was to transfer to another residency. More than half of these residents transferred into a different specialty, with a majority choosing nonsurgical specialties. Additionally, a majority of attrition occurred in PGY-2 or before. Sex was also observed to have a significant influence on attrition, with female residents having higher rates of attrition and more often leaving the field of neurosurgery. Finally, older age at the start of residency was found to be a significant factor in attrition. Future studies should emphasize targeted interventions at negating resident attrition, either by better educating candidates about the realities of neurosurgical training or by providing more comprehensive support to at-risk neurosurgical residents.

\section{Acknowledgments}

We would like to thank Yue-Fang Chang, $\mathrm{PhD}$, for her invaluable assistance with statistical analysis of the data.

\section{References}

1. Andriole DA, Jeffe DB, Hageman HL, Klingensmith ME, McAlister RP, Whelan AJ: Attrition during graduate medical education: medical school perspective. Arch Surg 143:11721177, 2008

2. Balch CM, Freischlag JA, Shanafelt TD: Stress and burnout among surgeons: understanding and managing the syndrome and avoiding the adverse consequences. Arch Surg 144:371376,2009

3. Bauer JM, Holt GE: National orthopedic residency attrition: who is at risk? J Surg Educ 73:852-857, 2016

4. Baxter N, Cohen R, McLeod R: The impact of gender on the choice of surgery as a career. Am J Surg 172:373-376, 1996

5. Bongiovanni T, Yeo H, Sosa JA, Yoo PS, Long T, Rosenthal M, et al: Attrition from surgical residency training: perspectives from those who left. Am J Surg 210:648-654, 2015

6. Dodson TF, Webb AL: Why do residents leave general surgery? The hidden problem in today's programs. Curr Surg 62:128-131, 2005

7. Dorsey ER, Jarjoura D, Rutecki GW: Influence of controllable lifestyle on recent trends in specialty choice by US medical students. JAMA 290:1173-1178, 2003

8. Everett CB, Helmer SD, Osland JS, Smith RS: General surgery resident attrition and the 80-hour workweek. Am J Surg 194:751-757, 2007

9. Gelfand DV, Podnos YD, Wilson SE, Cooke J, Williams RA: Choosing general surgery: insights into career choices of current medical students. Arch Surg 137:941-947, 2002

10. Kennedy KA, Brennan MC, Rayburn WF, Brotherton SE: Attrition rates between residents in obstetrics and gynecology and other clinical specialties, 2000-2009. J Grad Med Educ 5:267-271, 2013

11. Khoushhal Z, Hussain MA, Greco E, Mamdani M, Verma S, Rotstein $\mathrm{O}$, et al: Prevalence and causes of attrition among surgical residents: a systematic review and meta-analysis. JAMA Surg 152:265-272, 2017

12. Kwakwa F, Jonasson O: Attrition in graduate surgical education: an analysis of the 1993 entering cohort of surgical residents. J Am Coll Surg 189:602-610, 1999

13. Leibrandt TJ, Pezzi CM, Fassler SA, Reilly EF, Morris JB: Has the 80-hour work week had an impact on voluntary attrition in general surgery residency programs? J Am Coll Surg 202:340-344, 2006

14. Longo WE: Attrition: our biggest continuing challenge. Am J Surg 194:567-575, 2007

15. Manriquez Gilpin M: Residency attrition rate in obstetrics and gynecology: are we losing more postgraduates today? Am J Obstet Gynecol 193:1804-1806, 2005

16. Nadeem M, Effendi MS, Hammad Ather M: Attrition in surgical residency programmes: causes and effects. Arab J Urol 12:25-29, 2014

17. Naylor RA, Reisch JS, Valentine RJ: Factors related to attrition in surgery residency based on application data. Arch Surg 143:647-652, 2008

18. Nora LM, McLaughlin MA, Fosson SE, Stratton TD, Murphy-Spencer A, Fincher RM, et al: Gender discrimination and sexual harassment in medical education: perspectives gained by a 14-school study. Acad Med 77:1226-1234, 2002

19. Renfrow JJ, Rodriguez A, Liu A, Pilitsis JG, Samadani U, Ganju A, et al: Positive trends in neurosurgery enrollment and attrition: analysis of the 2000-2009 female neurosurgery resident cohort. J Neurosurg 124:834-839, 2016

20. Richardson HC, Redfern N: Why do women reject surgical careers? Ann R Coll Surg Engl 82 (9 Suppl):290-293, 2000

21. Stratton TD, McLaughlin MA, Witte FM, Fosson SE, Nora LM: Does students' exposure to gender discrimination and sexual harassment in medical school affect specialty choice and residency program selection? Acad Med 80:400-408, 2005

22. Sullivan MC, Yeo H, Roman SA, Ciarleglio MM, Cong X, Bell RH Jr, et al: Surgical residency and attrition: defining the individual and programmatic factors predictive of trainee losses. J Am Coll Surg 216:461-471, 2013

23. Wynn BO, Smalley R, Cordasco KM: Does it cost more to train residents or to replace them?: a look at the costs and benefits of operating graduate medical education programs. Rand Health Q 3:7, 2013

24. Zuccato JA, Kulkarni AV: The impact of early medical school surgical exposure on interest in neurosurgery. Can J Neurol Sci 43:410-416, 2016

\section{Disclosures}

The authors report no conflict of interest concerning the materials and methods used in this study or the findings specified in this paper.

\section{Author Contributions}

Conception and design: Agarwal, Chambless. Acquisition of data: White. Analysis and interpretation of data: White, Chambless. Drafting the article: Agarwal, White. Critically revising the article: Agarwal, Pannullo, Chambless. Reviewed submitted version of manuscript: all authors. Approved the final version of the manuscript on behalf of all authors: Agarwal. Statistical analysis: White. Administrative/technical/material support: Chambless. Study supervision: Chambless.

\section{Correspondence}

Nitin Agarwal: University of Pittsburgh Medical Center, Pittsburgh,PA. agarwaln@upmc.edu. 